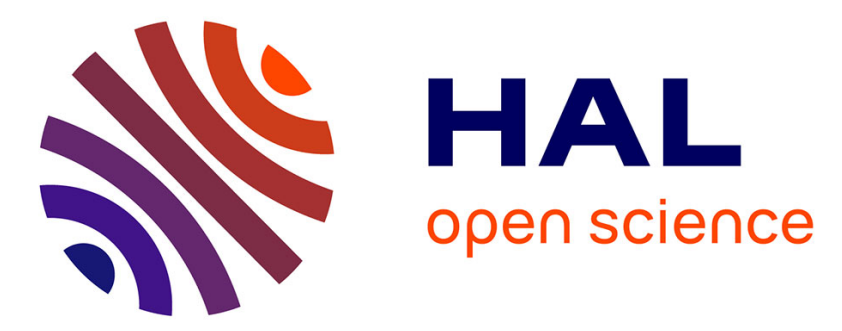

\title{
LAGEOS asymmetric reflectivity and corner cube reflectors
}

\author{
D. Vokrouhlicky, G. Métris
}

\section{To cite this version:}

D. Vokrouhlicky, G. Métris. LAGEOS asymmetric reflectivity and corner cube reflectors. Journal of Geophysical Research: Solid Earth, 2004, 109, pp.B10401. 10.1029/2003JB002921 . hal-00419018

\section{HAL Id: hal-00419018 \\ https://hal.science/hal-00419018}

Submitted on 10 Feb 2021

HAL is a multi-disciplinary open access archive for the deposit and dissemination of scientific research documents, whether they are published or not. The documents may come from teaching and research institutions in France or abroad, or from public or private research centers.
L'archive ouverte pluridisciplinaire HAL, est destinée au dépôt et à la diffusion de documents scientifiques de niveau recherche, publiés ou non, émanant des établissements d'enseignement et de recherche français ou étrangers, des laboratoires publics ou privés. 


\title{
LAGEOS asymmetric reflectivity and corner cube reflectors
}

\author{
D. Vokrouhlický \\ Institute of Astronomy, Charles University, Prague, Czech Republic \\ G. Métris \\ Observatoire de la Côte d'Azur, Department GEMINI, Grasse, France \\ Received 2 December 2003; revised 16 April 2004; accepted 1 July 2004; published 2 October 2004.
}

[1] Accurate laser tracking of LAGEOS satellites, allows investigation together with gravitational effects, a number of fine nongravitational perturbations in their orbit. Several of these forces are reasonably interpreted in terms of known physical phenomena. Postulated asymmetry in reflectivity of LAGEOS hemispheres is an exception. Here we show that in spite of a recent suggestion, this empirical effect cannot be explained by differential sunlight reflection on germanium and fused silica corner cube reflectors. The true nature of this effect remains puzzling. INDEX TERMS: 1241 Geodesy and Gravity: Satellite orbits; 1299 Geodesy and Gravity: General or miscellaneous; 3210 Mathematical Geophysics: Modeling; KEYWORDS: LAGEOS, nongravitational perturbations, optical anisotropy

Citation: Vokrouhlický, D., and G. Métris (2004), LAGEOS asymmetric reflectivity and corner cube reflectors, J. Geophys. Res., 109, B10401, doi:10.1029/2003JB002921.

\section{Introduction}

[2] Orbit analysis of the twin LAGEOS satellites is a remarkably difficult task principally because of their accurate observation data spanning a long period of time. It provides a superb characterization of the geopotential, including its long-period and irregular variations [e.g., Chen et al., 1999; Cox and Chao, 2002], plate tectonics [e.g., Dunn et al., 1990; Altamimi et al., 2002] and the Earth orientation parameters [e.g., Watkins and Eanes, 1994]. It became also a splendid opportunity to test relativistic effects [e.g., Ries et al., 1988], in particular, the Lense-Thirring vectorial component of the post-Newtonian gravitation [e.g., Ciufolini et al., 1998]. Additionally, after Smith and Dunn's [1980] discovery of a slow decrease of LAGEOS semimajor axis, it also allows/requires to study fine nongravitational effects [e.g., Rubincam, 1987, 1988; Afonso et al., 1989; Scharroo et al., 1991; Vokrouhlický and Farinella, 1995; Métris et al., 1997; Slabinski, 1997]. A possibility to study various nongravitational effects in LAGEOS orbits is an interesting scientific problem as such, but because of their characteristic indeterminism they also appear as a hindrance for understanding further details of both gravitational and relativity effects [e.g., Métris et al., 1997]. So a capability to remove uncertainties in their modeling is a very important task.

[3] The most puzzling nongravitational perturbation in LAGEOS orbit is the assumed asymmetry in reflectivity of the spacecraft hemispheres. This idea arose in the late 1980s and became gradually a part of LAGEOS literature (see a nice review by Rubincam [1993]). Here we use the work of Scharroo et al. [1991], who employed this

Copyright 2004 by the American Geophysical Union. 0148-0227/04/2003JB002921\$09.00 effect when attempting to fit the early series of the LAGEOS anomalous along-track acceleration. They showed that a difference $\Delta \rho$ in specular reflectivity of the Northern and Southern Hemispheres produces an acceleration

$$
\mathbf{f}_{A}=-\Phi \Delta \rho \sin ^{2} \theta_{r} \mathbf{s}
$$

along the direction $\mathbf{s}$ of the spin axis. Here, $\Phi=\pi R^{2} F /$ $(4 m c) \simeq 797 \mathrm{pm} / \mathrm{s}^{2}$, with $R$ the satellite radius, $m$ its mass, $c$ the light velocity and $F$ the solar radiation flux, and cos $\theta_{r}=\mathbf{s} \cdot \mathbf{n}_{0}$, with $\mathbf{n}_{0}$ unitary vector toward the Sun (so that $\theta_{r}$ is the angle between $\mathbf{s}$ and $\mathbf{n}_{0}$ ). A similar result was obtained by Slabinski [1997], who assumed hemispheric asymmetry of both specular and diffuse reflectivity. Note that Métris et al. [1997] made an error in reproducing equation (1), so that their formula (3) should read as equation (1) above. With $\Delta \rho \simeq-0.015$ and $\theta_{r} \simeq 90^{\circ}$, both appropriate for the initial 20 years of LAGEOS (Figure 1), the asymmetric reflectivity is basically an acceleration along the spacecraft spin axis with magnitude of $\simeq 12 \mathrm{pm} / \mathrm{s}^{2}$. It should be pointed out that the primary need to introduce the asymmetric reflectivity for LAGEOS orbits is to fit the anomalous along-track signal, where it contributes equally (or more) as the Yarkovsky-Schach effect during the periods when the satellite orbit enters the Earth's shadow. Though not entirely negligible, the asymmetric reflectivity contribution to the eccentricity vector excitation is minor, about an order of magnitude smaller than the signal produced by the Yarkovsky-Schach effect and a slight net recalibration of the surface reflectivity [Métris et al., 1997]. Thus a validation of any physical model aiming to explain the observed asymmetric reflectivity must focus on the observed along-track excitations rather than eccentricity vector excitations. 

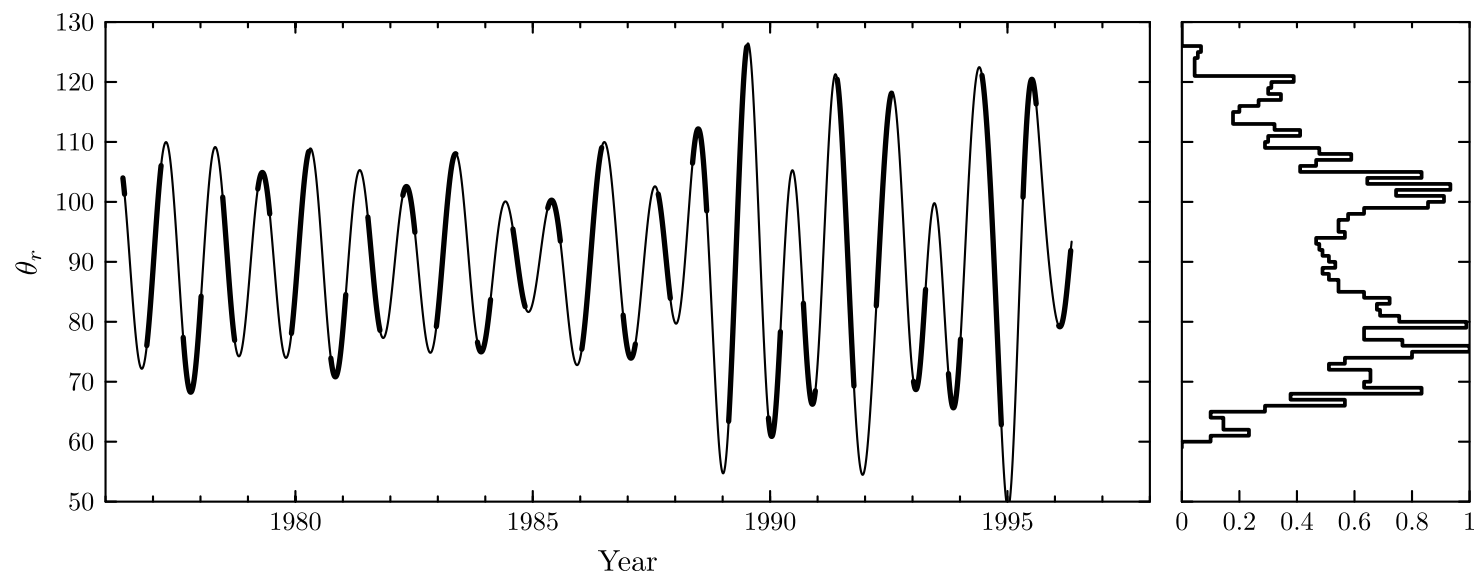

Figure 1. (left) Tilt angle $\theta_{r}$ between the solar direction $\mathbf{n}_{0}$ and the spin axis $\mathbf{s}$ of LAGEOS satellite as a function of time during the first 20 years of the mission (solution due to Bertotti and Iess [1991] and Farinella et al. [1996]). Thick intervals indicate periods of time when the satellite orbit crosses Earth's shadow. (right) Distribution of $\theta_{r}$ values, arbitrarily normalized to unity, when LAGEOS resides in Earth's shadow during the first 20 years of its mission.

[4] Recently, Lucchesi [2003, 2004] argued that the asymmetric reflectivity effect for LAGEOS satellites is understood in terms of sunlight reflection on germanium corner cube reflectors (CCRs). Here we however demonstrate that the solar reflection on the entire net of CCRs on the satellite surface, including the four germanium elements with different reflectivity features, amounts barely to about one third of the observed effect. Solar radiation reflection on CCRs thus cannot explain the empirical asymmetric reflectivity effect that remains a puzzling element in the theory of nongravitational forces affecting LAGEOS orbits. The most plausible cause, notably a possible small damage or pollution of one hemisphere during the satellite orbit insertion phase, has been discussed by Rubincam [1993] (who apparently arrived at a similar conclusion as obtained in this paper but did not publish his result).

\section{Theory}

[5] In order to accurately formulate recoil due to sunlight reflection on the spacecraft, we first consider a surface element $\Delta S$ with normal vector $\mathbf{n}$, such as the front facet of CCR. Assuming partial specular reflection, fractionally $\rho$ part of the incident radiation, and partial diffuse reflection, fractionally $a$ part of the incident radiation, sunlight exerts on the satellite the following force per unit of mass [Milani et al., 1987; Slabinski, 1997]:

$$
\Delta \mathbf{f}=-\Delta \Phi \cos \theta_{0}\left[(1-\rho) \mathbf{n}_{0}+2\left(\rho \cos \theta_{0}+a / 3\right) \mathbf{n}\right],
$$

with $\Delta \Phi=F \Delta S /(m c)$ and $\cos \theta_{0}=\mathbf{n} \cdot \mathbf{n}_{0}$. CCRs have a partial capability to reflect radiation backward; we shall denote $\rho^{\prime}$ the corresponding fraction, so that the backward mode of reflection contributes to the total recoil on the satellite with

$$
\Delta \mathbf{f}^{\prime}=-\Delta \Phi \rho^{\prime} \cos \theta_{0} \mathbf{n}_{0} .
$$

We now aim to obtain a supplementary force $\Delta \mathbf{f}_{T}$ per unit of mass that should be considered for explaining the anomalous orbital excitations of LAGEOS. For that purpose we must subtract the values of specular $\bar{\rho}$ and diffuse $\bar{a}$ reflectivity coefficients used in the background orbit determination model (those determining the radiation pressure coefficient $C_{R}$ ). Only this differential effect should correspond to the new perturbation to be considered. We thus obtain

$\Delta \mathbf{f}_{T}=-\Delta \Phi \cos \theta_{0}\left[\left(\bar{\rho}+\rho^{\prime}-\rho\right) \mathbf{n}_{0}+2\left[(\rho-\bar{\rho}) \cos \theta_{0}+(a-\bar{a}) / 3\right] \mathbf{n}\right]$.

In general, the reflectivity parameters $\rho, \rho^{\prime}$ and $a$ depend on the incidence angle $\theta_{0}$; this is particularly true for the backward component $\rho^{\prime}$ and specular $\rho$ components. In what follows we shall assume a simple five-parameter model

$$
\begin{gathered}
\rho\left(\theta_{0}\right)=\alpha-\beta \cos ^{k} \theta_{0}, \\
\rho^{\prime}\left(\theta_{0}\right)=(\gamma-\beta) \cos ^{k} \theta_{0},
\end{gathered}
$$

with $(\alpha, \beta, \gamma ; a)$ constants and $k$ an integer exponent. Note the case of specular reflection of the unpolarized light from a flat surface is traditionally treated using the Fresnel formulae [e.g., Born and Wolf, 1964], whose algebraic dependence on $\theta_{0}$ is too complicated to allow analytic analysis; however, equation (5a) represents an admittedly correct approximation for our purpose. Even more complex is the analysis of the retroreflected part for which equation (5b) is an approximation.

[6] The instantaneous acceleration equation (4) must be averaged over the satellite's rotation cycle, short compared to the relevant orbital timescales. This is a fairly standard procedure and we give only the final result (overbar indicates the rotation averaging)

$$
\overline{\Delta \mathbf{f}}_{T}=-\Delta \Phi\left(\mathcal{A} \mathbf{n}_{0}+\mathcal{B} \mathbf{s}\right) .
$$

As expected, $\overline{\Delta \mathbf{f}}_{T}$ has a component $(-\Delta \Phi \mathcal{A})$ along the solar direction $\mathbf{n}_{0}$, whose average is effectively included in the 
mean value of the radiation coefficient $C_{R}$, and a component $(-\Delta \Phi \mathcal{B})$ along the direction $\mathbf{s}$ of spacecraft spin axis. The latter component is of more importance here, since it seems to bear characteristics of the empirical optical asymmetry effect. Variations of the former component produce shortterm fluctuations of $C_{R}$. With a little algebra we obtained

$$
\mathcal{A}=\alpha^{\prime} \mathcal{I}_{1}+\gamma \mathcal{I}_{k+1}-2 \frac{\cos \delta}{\sin \theta_{r}}\left(\alpha^{\prime} \mathcal{J}_{2}+\beta \mathcal{J}_{k+2}+\varepsilon \mathcal{J}_{1}\right)
$$

$$
\begin{aligned}
\mathcal{B}= & -2 \sin \delta\left(\alpha^{\prime} \mathcal{I}_{2}+\beta \mathcal{I}_{k+2}+\varepsilon \mathcal{I}_{1}\right) \\
& +2 \cos \delta \frac{\cos \theta_{r}}{\sin \theta_{r}}\left(\alpha^{\prime} \mathcal{J}_{2}+\beta \mathcal{J}_{k+2}+\varepsilon \mathcal{J}_{1}\right),
\end{aligned}
$$

where $\varepsilon=(\bar{a}-a) / 3, \alpha^{\prime}=\bar{\rho}-\alpha, \delta$ is CCR's latitude with respect to the spacecraft "equator" and the $\mathcal{I}$ and $\mathcal{J}$ functions follow from recurrence series $(n \geq 0)$

$$
\mathcal{I}_{n+1}=A \mathcal{I}_{n}+B \mathcal{J}_{n},
$$

$\mathcal{J}_{n+1}=\frac{1}{n+2}\left[\frac{\sin \lambda_{0}}{\pi}\left(A+B \cos \lambda_{0}\right)^{n+1}+(n+1)\left(A \mathcal{J}_{n}+B \mathcal{I}_{n}\right)\right]$,

with the starting values

$$
\begin{gathered}
\mathcal{I}_{1}=\frac{1}{\pi}\left(A \lambda_{0}+B \sin \lambda_{0}\right), \\
\mathcal{J}_{1}=\frac{1}{2 \pi}\left[B \lambda_{0}+\sin \lambda_{0}\left(2 A+B \cos \lambda_{0}\right)\right] ;
\end{gathered}
$$

we use $A=\sin \delta \cos \theta_{r}, B=\cos \delta \sin \theta_{r}$,

$$
\cos \lambda_{0}= \begin{cases}-1, & \text { for } \delta>\theta_{r} \\ -\frac{\sin \delta}{\cos \delta} \frac{\cos \theta_{r}}{\sin \theta_{r}}, & \text { for }-\theta_{r}<\delta<\theta_{r} \\ 1, & \text { for } \delta<-\theta_{r}\end{cases}
$$

for $\theta_{r}<\pi / 2$ and

$$
\cos \lambda_{0}= \begin{cases}-1, & \text { for } \delta<\theta_{r}-\pi \\ -\frac{\sin \delta}{\cos \delta} \frac{\cos \theta_{r}}{\sin \theta_{r}}, & \text { for } \theta_{r}-\pi<\delta<\pi-\theta_{r} \\ 1, & \text { for } \delta>\pi-\theta_{r}\end{cases}
$$

for $\theta_{r}>\pi / 2$. We do not give here a more simple, but equally straightforward, formulae for polar CCRs $\left(\delta= \pm 90^{\circ}\right)$.

[7] In the last step, a contribution from all surface CCRs should be combined to obtain the final anomalous acceleration due to fine details of sunlight reflection on the satellite surface; distribution of CCRs on LAGEOS surface and their surface area $\Delta S \simeq 11.5 \mathrm{~cm}^{2}$ are taken from Avizonis [1997] [see also Johnson et al., 1976; Cohen and Smith, 1985;
Slabinski, 1997]. The suggested source of the optical anisotropy effect by Lucchesi [2003, 2004] stems from observation, that of the 426 CCRs on the LAGEOS satellite four are made of germanium (to facilitate laser ranging experiments with infrared systems), while the remaining 422 are made of fused silica. The front surface reflectivity of the germanium CCRs is significantly higher than those of the fused silica CCRs; indeed, LAGEOS 1 photometry reported by Avizonis [1997] demonstrates sunlight reflections from germanium CCRs appear about twice as bright as those from fused silica CCRs. Avizonis [1997] thus characterizes their respective ability to reflect unpolarized light as media with refractive indices $n \simeq 4$ (for the germanium CCRs) and $n \simeq 1.5$ (for the fused silica CCRs), though certainly this is a crude approximation and needs to be substantiated with experimental data. In the LAGEOS 1 case, the four germanium CCRs are located asymmetrically with respect to the spacecraft equator, namely one on the northern pole and three equally spaced in longitude along the $-22.98^{\circ}$ latitude band (e.g., Johnson et al. [1976], Cohen and Smith [1985], Avizonis [1997], or Lucchesi [2004]). In the LAGEOS 2 case, the germanium CCRs are located symmetrically with respect to the spacecraft equator at $\pm 31.23^{\circ}$ latitudes [e.g., Lucchesi, 2004].

[8] In what follows, we examine whether the resulting acceleration component along the spin axis, $-\Delta \Phi \Sigma_{\mathrm{CCR}} \mathcal{B}(\delta$, $\theta_{r}$ ), can explain the required optical asymmetry effect (equation (1)). We restrict our analysis to the case of LAGEOS 1 satellite, but our conclusion should apply to the case of LAGEOS 2 as well.

\section{Case of LAGEOS 1}

[9] Since $\mathbf{f}_{A}$ and $\overline{\Delta \mathbf{f}}_{T}$ change with time only via spacecraft spin axis tilt from the solar direction denoted $\theta_{r}$, we first determine the appropriate range of values attained by this angle (Figure 1). We restrict our analysis to the first $20 \mathrm{yr}$ of LAGEOS mission, during which we have a reliable enough theoretical model of LAGEOS's spin axis evolution (see Vokrouhlický [1996] and Métris et al. [1999] for comments). Given the purpose of our study this limitation is not important. We note the near polar direction of the LAGEOS spin axis implies $\theta_{r}$ stays constrained within some interval near $90^{\circ}$, and its variations are mainly due to the ecliptic inclination with respect to the equator. The larger amplitude in the 1990s is due to the onset of regular precession of the spacecraft spin axis after the gravitational torque start to dominate the magnetic torque [Bertotti and Iess, 1991; Farinella et al., 1996]. Figure 1 (right) shows statistical distribution of $\theta_{r}$ values recorded when the LAGEOS orbit was crossing the Earth's shadow, notably when the asymmetric reflectivity effect contributes to the anomalous alongtrack orbital perturbation.

[10] With this information, we conducted the following test. We randomly chose a large number of parametric sets $(\alpha, \beta, \gamma, \varepsilon, k)$, recall definition of $\varepsilon$ given after equation ( $7 \mathrm{~b})$, characterizing sunlight reflection on CCRs (we note the result depends on the assumed values of $\bar{a}$ and $\bar{\rho}$ only very weakly). Parameters for those made of germanium and fused silica were considered different. In each of these cases we only controlled obvious constraints such as the total reflectivity coefficient is not larger than unity. We 
performed a weighted correlation analysis of the amplitude $\left(f_{A}\left(\theta_{r}\right)=-\Phi \Delta \rho \sin ^{2} \theta_{r}\right)$ of the empirical model of the asymmetric reflectivity effect (fitted to the observations) and the prediction $\left(f_{B}\left(\theta_{r}\right)=-\Delta \Phi \Sigma_{\mathrm{CCR}} \mathcal{B}\left(\delta, \theta_{r}\right)\right)$ by our model of the sunlight reflection on CCRs (equation (6)). In quantitative terms, we computed the "weighted correlation function"

$$
r(\alpha, \beta, \gamma, \varepsilon, k)=\frac{\int d \theta_{r} w\left(\theta_{r}\right) f_{A}\left(\theta_{r}\right) f_{B}\left(\theta_{r}\right)}{\int d \theta_{r} w\left(\theta_{r}\right) f_{A}^{2}\left(\theta_{r}\right)}
$$

where $w\left(\theta_{r}\right)$ is the weight function of the $\theta_{r}$ occurrence from Figure 1. A CCR reflection solution with $r$ near unity would mean a model that reliably well explains the empirical asymmetric reflectivity effect. The maximum value of $r$ we were able to find by running a million test cases was $\simeq 0.35$ which means a fairly poor match of the two models. Note that an order of magnitude misfit between the two models corresponds to $r \simeq 0.1$, and that is approximately what happens for most of the realistic sets of parameters $(\alpha, \ldots, k)$. The amplitude of the along-axis acceleration predicted by the CCRs reflection model is always significantly smaller than the value needed to explain the anomalous along-track signal of LAGEOS (and given by the empirical model (equation (1)).

[11] Figure 2 shows a comparison of the along-axis acceleration of the highest- $r$ model (thick line 1) as compared with the empirical model (equation (1)) (dashed line). We also made the same analysis with a restricted model where we included information about the refraction index $n$ for the fused silica glass $(n \simeq 1.5)$ and for germanium $(n \simeq 4)$ [Avizonis, 1997]. That constrains specular reflectivity to near unity at grazing angles and $\simeq 0.04, \simeq 0.36$ respectively, at zero incidence angle for the two materials and consequently fixes values of $\alpha$ and $\beta$ in Equations (5). In particular we set $\alpha=1$ for both types of CCRs, while $\beta=$ 0.64 and $\beta=0.96$ for germanium and fused silica CCRs respectively. We then let $\gamma$ and $k$ change and sought the highest- $r$ solution within this restricted model. The result is shown by curve 2 in Figure 2. As expected with less degrees of freedom the empirical model misfit is still larger (we obtained maximum $r \simeq 0.22$ ).

[12] Finally, we also directly used our formula (6) for the sunlight pressure on CCRs to fit the anomalous along-track acceleration of LAGEOS 1 (data by R.J. Eanes and J.C. Ries were acquired through a public ftp site $\mathrm{ftp} / / \mathrm{ftp} . \mathrm{csr}$. utexas.edu/pub/slr at CSR/UT), following thus the work of Scharroo et al. [1991] and others. We first subtracted a constant drag of $1 \mathrm{pm} / \mathrm{s}^{2}$ (as given by charged drag), Yarkovsky thermal drag with amplitude of $3 \mathrm{pm} / \mathrm{s}^{2}$ and Yarkovsky-Schach effect with amplitude of $240 \mathrm{pm} / \mathrm{s}^{2}$ and phase lag $f_{0}=188^{\circ}$ [see Métris et al., 1997]. The residual signal was analyzed using the original Scharroo et al. [1991] optical asymmetry acceleration equation (1) and our model discussed in section 2. As expected, in the first case the best fit is achieved with $\Delta \rho=-0.015$; for that value the correlation of the residual along-track signal and that from the empirical asymmetry model is $\simeq 0.6$ (recall only observations till 1996 are taken into account). This indicates that the bulk of the remaining signal may be admittedly interpreted by the empirical model. However, trying to

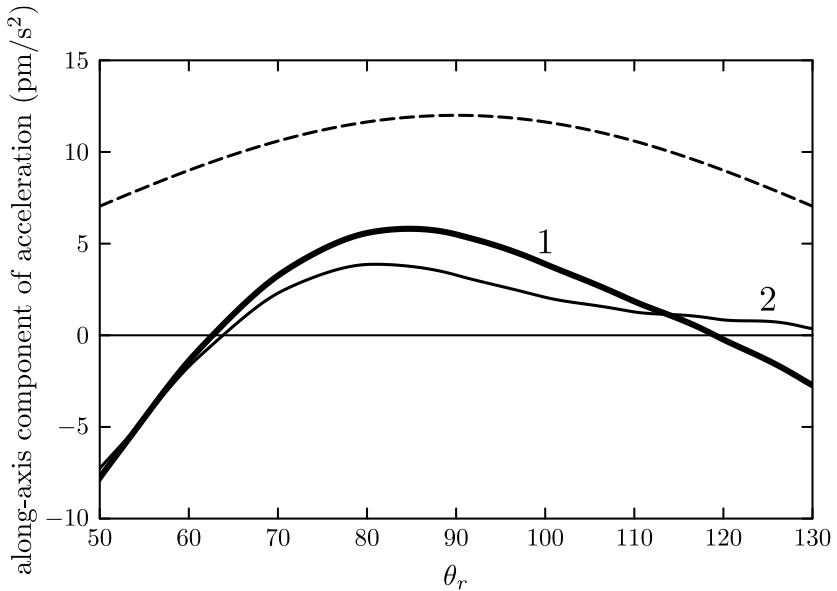

Figure 2. Along-axis acceleration component (in $\mathrm{pm} / \mathrm{s}^{2}$ ) as a function of the solar direction tilt angle $\theta_{r}$ for (1) empirical asymmetric reflectivity model (equation (1)), dashed line, and (2) our highest- $r$ model of sunlight reflection of LAGEOS CCRs, thick solid line labeled 1. The thinner solid line labeled 2 is also a maximum- $r$ model but for a restricted set of a parametric choice (see the text). Compare these results with Figure 3 of Lucchesi [2004].

explain the same residual signal using the parameterized model (equation (6)) the correlation dropped to a maximum value of $\simeq 0.13$ even with a million trial cases for CCR parameter sets. This again clearly shows insufficiency of the CCR reflection model to replace the optical asymmetry effect.

\section{Conclusions}

[13] Both quantitative tests in section 3 show the radiative recoil due to the sunlight reflection on CCRs amounts to less than one third of the observed optical anisotropy effect for LAGEOS 1. The qualitative basis of this mismatch is twofold: (i) CCRs are too numerous on LAGEOS surface so that they form a very regular pattern (hence approximating "true" sunlight reflection with a spherical model of constant reflectivity parameters is fairly satisfactory), and (ii) germanium CCRs are too few compared to the fused silica CCRs, to produce a significant asymmetry of the reflection.

[14] Note the amplitude of our results from Figure 2 is still larger than the estimated along-axis acceleration by Lucchesi [2004, Figure 3]. With that Lucchesi should have reached the same conclusion as here; however, an incorrect methodology in his paper, and also by Lucchesi [2003], led to an apparent positive solution. By considering radiation recoil from "ad hoc" added germanium CCRs, the principal contribution to the eccentricity vector was that along the solar direction $\mathbf{n}_{0}$. However, as discussed in section 2 , this part is effectively absorbed in the radiation pressure coefficient $C_{R}$ and should not be mislead for the optical asymmetry effect. Moreover, we recall the optical asymmetry effect's principal importance is to contribute to the observed along-track signal and not the eccentricity vector excitation [Métris et al., 1997].

[15] The asymmetric optical reflectivity remains a troubling element for the theory of nongravitational forces in 
LAGEOS orbits. This paper should stimulate more theoretical and laboratory work in recognizing its true nature.

[16] Acknowledgments. We thank E. C. Pavlis and D. P. Rubincam for their critical reviews that helped to improve the final version of the paper. The work of DV was supported by a research plan J13/98: 113200004 of the Czech Ministry of Education.

\section{References}

Afonso, G., et al. (1989), Orbital effects of LAGEOS seasons and eclipses, Ann. Geophys., 7, 501-514.

Altamimi, Z., C. Boucher, and P. Sillard (2002), New trends for realization of the International Terrestrial Reference System, Adv. Space Sci., 30, $175-184$.

Avizonis, P. V. (1997), Remote sensing of the LAGEOS-I spin-axis and image processing for advanced optical systems, Ph.D. thesis, Univ. of Maryland, College Park.

Bertotti, B., and L. Iess (1991), The rotation of LAGEOS, J. Geophys. Res., 96, 2431-2440

Born, M. and E. Wolf (1964), Principles of Optics, Pergamon, New York

Chen, J. L., C. R. Wilson, R. J. Eanes, and B. D. Tapley (1999), Geophysical contributions to satellite nodal residual variation, J. Geophys. Res. $104,23,237-23,244$

Ciufolini, I., E. Pavlis, F. Chieppa, E. Fernandes-Vieira, and J. Perez-Mercader (1998), Test of general relativity and measurement of the Lense-Thirring effect with two Earth's satellites, Science, 279, 2100-2103.

Cohen, S. C., and D. E. Smith (1985), LAGEOS scientific results: Introduction, J. Geophys. Res., 90, 9217-9220.

Cox, C. M., and B. F. Chao (2002), Detection of a large-scale mass redistribution in the terrestrial system since 1998, Science, 297, 831-833.

Dunn, P. J., J. W. Robbins, M. H. Torrence, S. M. Klosko, R. G. Williamson, E. C. Pavlis, N. C. Douglas, and S. K. Fricke (1990), Tectonic motion and deformation from satellite laser ranging to LAGEOS, J. Geophys. Res., 95, 22,013-22,041.

Farinella, P., D. Vokrouhlický, and F. Barlier (1996), The rotation of LAGEOS and its long-term semimajor axis decay: A self-consistent solution, J. Geophys. Res., 101, 17,861-17,872.

Johnson, C. W., C. A. Lundquist, and J. L. Zurasky (1976), The LAGEOS satellite, paper presented at the XXVIIth Congress, Int. Astron. Fed., Anaheim, Calif.

Lucchesi, D. M. (2003), The asymmetric reflectivity effect on the LAGEOS satellites and the germanium retroreflectors, Geophys. Res. Lett., 30(18), 1957, doi:10.1029/2003GL017741
Lucchesi, D. M. (2004), LAGEOS satellites germanium cube-corner retroreflectors and the asymmetric reflectivity effect, Celest. Mech. Dyn. Astron., 88, 269-291.

Métris, G., D. Vokrouhlický, J. C. Ries, and R. J. Eanes (1997), Nongravitational effects and the LAGEOS eccentricity excitations, J. Geophys. Res., 102, 2711-2729.

Métris, G., D. Vokrouhlický, J. C. Ries, and R. J. Eanes (1999), LAGEOS spin axis and non-gravitational excitations of its orbit, Adv. Space Res., $23,721-725$.

Milani, A., A. M. Nobili, and P. Farinella (1987), Non-gravitational Perturbations and Satellite Geodesy, A. Hilger, Bristol, U.K.

Ries, J. C., C. Huang, and M. M. Watkins (1988), Effect of general relativity on a near-Earth satellite in the geocentric and barycentric reference frames, Phys. Rev. Lett., 61, 903-906.

Rubincam, D. P. (1987), LAGEOS orbit decay due to infrared radiation from Earth, J. Geophys. Res., 92, 1287-1294.

Rubincam, D. P. (1988), Yarkovsky thermal drag on LAGEOS, J. Geophys. Res., 93, 13,805-13,810.

Rubincam, D. P. (1993), The LAGEOS along-track acceleration: A review, in Relativistic Gravitational Experiments in Space, edited by M. Demianski and C. W. F. Everitt, pp. 1995-210, World Sci., River Edge, N. J.

Scharroo, R., K. F. Wakker, B. A. C. Ambrosius, and R. Noomen (1991), On the along-track acceleration of the LAGEOS satellite, J. Geophys. Res., 96, 729-740.

Slabinski, V. J. (1997), A numerical solution for LAGEOS thermal thrust: The rapid-spin case, Celest. Mech. Dyn. Astron., 66, 131-179.

Smith, D. E., and P. J. Dunn (1980), Long term evolution of the LAGEOS orbit, Geophys. Res. Lett., 7, 437-440.

Vokrouhlický, D. (1996), Nongravitational effects and LAGEOS rotation, Geophys. Res. Lett., 23, 3079-3082.

Vokrouhlický, D., and P. Farinella (1995), Specular reflection of sunlight from wavy ocean surfaces and the albedo effect on satellite orbits. II. LAGEOS long-term albedo perturbations revisited, Astron. Astrophys., 301, 282-289.

Watkins, M. M., and R. J. Eanes (1994), Diurnal and semidiurnal variations in Earth orientation determined from LAGEOS laser ranging, J. Geophys. Res., 99, 18,073-18,079.

G. Métris, Observatoire de la Côte d'Azur, dept. GEMINI, Av. N. Copernic, F-06130 Grasse, France. (gilles.metris@obs-azur.fr)

D. Vokrouhlický, Institute of Astronomy, Charles University, V Holešovičkách 2, CZ-18000 Prague 8, Czech Republic. (vokrouhl@mbox. cesnet.cz) 